\title{
KERNFORSCHUNGSZENTRUM
}

\author{
KARLSRUHE
}

Institut für Experimentelle Kernphysik

Search of Parity Mixing in ${ }^{180}$ Hf by a Measurement of the Circular Polarization of $\gamma$ Rays

P. Bock, B. Jenschke, H. Schopper

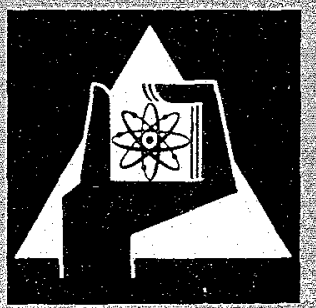

GESELSCHAFT HR KERNFORSCHUNG M. B. 


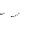




\section{PHYSICS LETTERS}

Volume 22, number 3,15 August 1966

SEARCH OF PARITY MIXING IN $180 \mathrm{HF}$ BY

A MEASUREMENT OF THE CIRCULAR POLARIZATION OF $\gamma$ RAYS

P. BOCK, B. JENSCHKE, H. SCHOPPER

pp. $316-317$

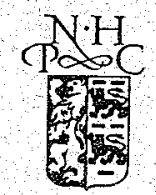

NORTH-HOLLAND PUBLISH ING COM PANY AMSTER D A M 



\title{
SEARCH OF PARITY MIXING IN $180_{\text {Hf }}$ BY A MEASUREMENT OF THE CIRCULAR POLARIZATION OF $\gamma$ RAYS
}

\author{
P.BOCK, B.JENSCHKE and H.SCHOPPER \\ Institut für Experimentelle Kermphysik der Technischen Hochschule \\ und des Kernforschungszentrums Karlsruhe, Germany
}

Received 15 July 1966

The circular polarization of the $57 \mathrm{keV}$ transition as well as of the $501 \mathrm{keV}$ cross-over transition was measured. Within experimental errors, no polarization was observed.

The current-current hypothesis [1] for the weak interaction implies a weak parity violating nucleon-nucleon force. A detection of the parity mixing in nuclear levels introduced by this force seems to be the only way to test this hypothesis experimentally with the facilities presently available. Several experiments have been performed with this aim [2]. Because of the extremely small effects to be observed these measurements are difficult and the results are partly at variance. Therefore it is desirable to find cases showing larger effects.

Scharff-Goldhaber and McKeown [3] have recently measured the internal-conversion coefficient of the highly hindered $57.6 \mathrm{keV}$ transition in $180 \mathrm{Hf}$ and find that no mixture of odd-parity multipolarities (E1, M2 etc.) would fit their data. Their results could be explained by a $90.5 \% \mathrm{E} 1+$ 9.5\% M1 transition indicating a strong parity mixture. An alternative interpretation is offered by the penetration effect which takes into account the influence of nuclear structure dependent terms on the internal conversion process. Indeed Hager and Seltzer [4] have shown that the anomalies of the measured conversion coefficients can be attributed to the penetration effect. However, quantitative calculations cannot be performed for the transition under consideration and hence an M1 admixture cannot be excluded.

In order to settle this question Goldhaber and McKeown have suggested to measure the circular polarization of the $57 \mathrm{keV} \gamma$ transition. A $9.5 \% \mathrm{M} 1$ admixture implies a polarization of $58 \%$. We have measured the circular polarization of the $57 \mathrm{keV}$ transition as well as of the $501 \mathrm{keV}$ cross-over transition. Within the experimental errors no polarization is observed, excluding a large parity admixture. To measure the circular polarization we used the Compton forward scattering from a magnetized iron cylinder [5]. Although in this case the analyzing efficiency is small for photon energies as low as $57 \mathrm{keV}$ it is higher than for any other known method. However, the background from other $\gamma$ transitions had to be avoided as far as possible. For this purpose the source material $\left(20 \mathrm{mg} / \mathrm{cm}^{2}\right)$ was enriched to $58 \%{ }^{179} \mathrm{Hf}$ before irradiation resulting in a $180 \mathrm{Hf}$ activity of $81 \%$ at the beginning of the measurements. In addition the iron scatterer was made only $0.2 \mathrm{~mm}$ thick in order to improve after the scattering the ratio between the $57 \mathrm{keV}$ photons and quanta with higher energies. In order to avoid the scattering from the magnetizing coil and the yoke an especially designed magnet has been used [6]. The detecting system consisted of a NaI-crystal $(2 \mathrm{~mm}$ thick, 2.0 inch diameter), a $35 \mathrm{~cm}$ long light guide and a magnetically shielded 56 AVP multiplier tube. As a result of these measures the $57 \mathrm{keV}$ line showed up clearly in the $\gamma$ spectrum after scattering with a background of about $30 \%$. This line was selected with a discriminator window. Two more windows were set on the lower and upper side of the line, respectively, and data were taken for all three discriminator channels simultaneously. In this way electronic instabilities and the influence of magnetic stray fields could be controlled continuously. The magnetization of the scattering cylinder was changed every $20 \mathrm{sec}$. The data were punched on tape and analyzed on a computer including statistical tests. The counting rate was of the order of $4 \times 10^{4}$ counts per second at the beginning of the measurements. The influence of the stray fields was measured between individual runs by allowing the quanta to impinge on the scintillator without scattering from the iron. The effect of the stray field was always smaller 
than $2 \times 10^{-5}$ and could be neglected.

From the relative change of counting rate $\delta$ on reversing the magnetization one obtains the polarization $P$ according to:

$$
\delta=2\left(N^{+}-N^{-}\right) /\left(N^{+}+N^{-}\right) \stackrel{2}{=} 2 f\left\langle\mathrm{~d} \sigma_{\mathrm{C}} / \mathrm{d} \sigma_{0}\right\rangle,
$$

where $f$ is the fraction of oriented electrons in the iron which was 0.056 in our case. The analyzing efficiency $\left\langle\mathrm{d} \sigma_{\mathrm{c}} / \mathrm{d} \sigma_{\mathrm{o}}\right\rangle$ averaged over the geometry was calculated with a computer program [7] with the result $\left\langle\mathrm{d} \sigma_{\mathrm{c}} / \mathrm{d} \sigma_{\mathrm{o}}\right\rangle=0.061$.

In order to check these calculations and to test the proper functioning of the equipment the polarization of the internal bremsstrahlung of ${ }^{32} \mathrm{P}$ was measured for photon energies around $60 \mathrm{keV}$. An effect $\delta=(1.7 \pm 0.3) \times 10^{-4}$ corresponding to a polarization of $P=(4.7 \pm 0.9) \%$ was found which has to be compared with an expected polarization of $6.7 \%$. This agreement is satisfactory taking into account that a quantitative evaluation of these measurements is difficult because of the continuous bremsstrahlung spectrum and an appreciable contribution from the external bremsstrahlung, whose polarization is considerably reduced because of the electron scattering.

The uncorrected result of our measurements for the $57 \mathrm{keV}$ transition is $\delta=(-0.29 \pm 0.39) \times 10^{-4}$ and from eq. (1) one infers

$$
P=(-2.3 \pm 3) \% \text {. }
$$

Here a correction of $58 \%$ for the $\mathrm{K}$ radiation of $180 \mathrm{Hf}$ which accidentally has the same energy as the $57 \mathrm{keV} \gamma$ transition, of $30 \%$ for higher $\gamma$ transitions and $36 \%$ for coherent scattering has been applied. Allowing for systematic errors one obtains the estimate

$$
P<5 \%
$$

as compared to $P \approx 58 \%$ for a parity mixing.

The polarization of the $501 \mathrm{keV}$ cross-over transition was measured with the equipment used previously to investigate the decay of $181 \mathrm{Ta}$ [2]. An effect $\delta=(8.3 \pm 5.2) \times 10^{-5}$ was observed.

$$
P=(-1.4 \pm 0.9) \%
$$

after corrections for other $\gamma$-transitions.

The fact that no parity mixing is observed for the $180 \mathrm{Hf}$ decays does not contradict the currentcurrent hypothesis. Lawson and Segel [8] have shown that the $\mathrm{K}$ selection rule which inhibits the $E 1$ radiation also affects the parity-forbidden $M 1$ transition resulting in a very small polarization.

\section{References}

1. R.P.Feynman and M.Gell-Mann, Phys. Rev.109 (1958) 193;

M. Gell-Mann, Rev. Mod. Phys. 31 (1959) 831.

2. F.Boehm and E. Kankeleit, Phys. Rev. Letters 11 (1965) 312;

Y.G.Abov, P.A.Krupchitsky and Y.A. Oratovsky, Physics Letters 12 (1964) 25;

P.Bock and H.Schopper, Physics Letters 16 (1965) 281 ;

M. Forte and O. Saavedra, Proc. Karlsruhe Conf. on polarization phenomena, Birkhäuser, Basel (1966); W. M. Lobashov, W. L. Nașarenko, L. F. Sajenko,

L.M. Smotritzki, Pisma JETP 3 (1966) 76, 268.

3. G. Scharff-Goldhaber and M. McKeown, Indication of Parity Mixing in an Electromagnetic Transition, Intern. Conf. on Weak Interaction, Chicago, October 1965, ANL-Report 7130.

4. R.Hager and E. Seltzer, Physics Letters 20 (1966) 180.

5. H. Schopper, Nucl. Instr. 3 (1958) 158.

6. H. Huber, S. Galster and H. Schopper, Nucl. Instr. Methods 21 (1963) 338.

7. H. Behrens, Thesis Karlsruhe (1966).

8. R.D. Lawson and R. E.Sege1, Phys, Rev. Letters 16 (1966) 1006. 